Check for updates

Cite this: RSC Adv., 2018, 8, 32009

Received 2nd August 2018

Accepted 9th September 2018

DOI: $10.1039 / c 8 r a 06498 a$

rsc.li/rsc-advances

\section{Ultrasensitive fluorescent aptasensor for MUC1 detection based on deoxyribonuclease I-aided target recycling signal amplification $\uparrow$}

\author{
Jun Zhang,,$_{t^{a}}$ Fengying Ran, $\neq^{a}$ Wenbo Zhou, ${ }^{a}$ Bing Shang, ${ }^{a}$ Fei $Y u,{ }^{a}$ Lun Wu, ${ }^{a}$ \\ Wanbao $\mathrm{Hu}^{\mathrm{b}}$ Xueqin $\mathrm{He}^{\mathrm{a}}$ and Qinhua Chen (iD) *a
}

\begin{abstract}
A novel sensing strategy for sensitive detection of mucin 1 protein (MUC1) based on deoxyribonuclease Iaided target recycling signal amplification was proposed. In this paper, in the absence of MUC1, the MUC1 aptamer is absorbed on the surface of graphene oxide (GO) via $\pi$-stacking interactions. This results in quenching of the fluorescent label and no fluorescence signal is observed. Upon adding MUC1, the probe sequences could be specifically recognized by MUC1, leading to an increase in the fluorescence intensity. The detection limit is as low as $10 \mathrm{pg} \mathrm{mL}^{-1}$, and a linear range from $50 \mathrm{pg} \mathrm{mL}^{-1}$ to $100 \mathrm{ng}$ $\mathrm{mL}^{-1}$. The assay is specific and sensitive, and successfully applied to the determination of MUC1 in spiked human serum, urine and saliva. Importantly, the proposed aptasensing strategy has great potential in detecting various protein and even cancer cells.
\end{abstract}

\section{Introduction}

At present, cancer is one of the most life-threatening diseases in the worldwide. ${ }^{1}$ The latest WHO figures show that 8.8 million people worldwide die from cancer every year, accounting for nearly $1 / 6$ of deaths a year, and the figure is expected to increase to more than 21 million by 2030 . Thus, it is important to develope effective methods for cancer therapy. Among them, the early identification and quantitative analysis of tumor biomarkers could provide a simpler and more effective method for monitoring tumor progression. It is of particular importance in early clinical diagnosis and treatment of cancer.

The mucin1 protein (MUC1), is a cell-surface associated glycoprotein of the mucin family, ${ }^{2}$ which contains a hydrophobic membrane-spanning domain of 31 amino acids, a cytoplasmic domain of 69 amino acids, and an extracellular domain consisting of a region of nearly identical repeats of 20 amino acids per repeat. ${ }^{3,4}$ MUC1 presents in a variety of malignant tumors and is over-expressed in almost all human epithelial, including breast, gastric, colorectal, lung, prostate, ovarian, pancreatic and bladder carcinomas; ${ }^{5}$ but, low levels or no expression in normal healthy tissues in normal cells. It is considered as a potential cancer biomarker. Thus, an sensitive detection of MUC1 is great importance in preliminary diagnosis

${ }^{a}$ Affiliated Dongfeng Hospital, Hubei University of Medicine, Shiyan, Hubei, 442008, China. E-mail: cqh77@163.com; Tel: +860719-8272283

${ }^{b}$ Sinopharm Dongfeng Huaguo Hospital, Shiyan 442008, Hubei, China

$\dagger$ Electronic supplementary information (ESI) available. See DOI: 10.1039/c8ra06498a

¥ Jun Zhang and Fengying Ran are all the first authors. of cancer. To date, several techniques including ELISA, ${ }^{6}$ surface plasmon resonance spectroscopy (SPR), ${ }^{7}$ colorimetric, ${ }^{8}$ electrochemistry, ${ }^{9-11}$ photoelectrochemical, ${ }^{12}$ electrochemical biosensors, ${ }^{13-16}$ electrochemiluminescence ${ }^{17-19}$ and fluorescence biosensors $^{20-23}$ have been applied in the detection of MUC1. Among them, fluorescence assay is a well-known detection method with acknowledged advantages such as high sensitivity, easy readout, simplicity and the feasibility of quantification. ${ }^{22,24}$ Despite many advances in this field, these strategies still have shortcomings including the costly modification of fluorophores and potential false positive signals. Moreover, the sensitivity needs to be further improved. Therefore, it's important to develop an efficient and highly sensitive fluorescent strategy to detect MUC1 in the fundamental research and practical applications.

In recent years, there have some reports of mucin 1 glycoprotein based on MUC-1 aptamer. Aptamers are nucleic acid ligands (DNA or RNA) that can specifically bind protein, which are artificially synthesized and selected in vitro through exponential enrichment (SELEX). ${ }^{25,26}$ In comparison with other targeting agents, aptamers possess unique advantages such as small size, design flexibility, stability in harsh biological environments, easy for chemical modification as well as high specificity and high purity. ${ }^{27,28}$ These special characteristics will enable them be widely used biomarker discovery and biosensors. ${ }^{29,30}$ To date, a series of aptamer-based biosensors have been applied to detect VEGF $165,{ }^{31}$ EpCAM,${ }^{32}$ Salmonella paratyphi $\mathrm{A}^{33}$ cocaine ${ }^{34}$ and so on. There have also been reported for detecting MUC1 using electrochemical, ${ }^{35}$ photoelectrochemical, ${ }^{12}$ colorimetric ${ }^{8}$ and fluorescent. ${ }^{22}$ But, among them, no standard method has been established. Therefore, 
a novel, highly sensitive and selective approaches for MUC1 detection need to be designed.

Graphene oxide (GO), a one-atom-thick two-dimensional carbon nanomaterial with a honeycomb structure. It's owing to unique features such as high mechanic strength, good water dispersibility and facile surface modification. ${ }^{36,37}$ GO has a high specific surface are a for binding plenty of molecules, such as nucleic acids, proteins, liposomes and pharmaceutical molecules. ${ }^{38-40}$ Moreover, GO is capable of quenching fluorescence with high efficiency, and can be used to construct GObased sensors based on the principle of fluorescence resonance energy transfer (FRET). ${ }^{41}$ More importantly, GO can improve their stability in the biological environments, due to its protection capacity for biomolecules binded to its surface from enzymatic cleavage. ${ }^{42}$ Consequently, GO, as a superb nanomaterial, would be easily used in biosensors for biochemical analysis. On the other hand, to overcome these limitations, signal amplification strategies, enzyme-aided signal amplification have recently been developed to achieve the sensitive detection of biomolecules, including endonuclease, deoxyribonuclease I (DNase I), exdonuclease and DNA polymerase. ${ }^{43}$ Among them, DNase I, a common endonuclease, can digest single- and double-stranded DNA (dsDNA) into oligo- and mononucleotides. DNase I can cleave phosphodiester bonds to product many polynucleotides with $5^{\prime}$ phosphate and $3^{\prime}-\mathrm{OH}$ groups, but cannot digest RNA sequences. Thus, these makes it suitable for RNA analysis based on DNase I-assisted target recycling and signal amplification. ${ }^{44}$ Based on these findings, He et al., described a approach nanographite-based fluorescent biosensor and DNase I for the amplified detection of Salmonella enteritidis, ${ }^{\mathbf{4 3}}$ and Yan et al., described a highly sensitive fluorescent aptasensor for Salmonella paratyphi A via DNase I-mediated cyclic signal amplification ${ }^{33}$ and so on. DNase I-aided signal amplification has the advantages of sensitive, reliable and specific. To meet the demands of the specificity and sensitivity of MUC1 detection, the development of an DNase I-aided signal amplification strategy is extremely urgent.

Hence, in this work, a very sensitive and specific aptasensor was designed to detect MUC1 based on DNase I-aided target recycling signal amplification. The MUC1 aptamer $5^{\prime}$ terminal was labeled with an FAM that amplifies fluorescence signals by cleaving the aptamer randomly. In the absence of MUC1, the MUC1 aptamer is strongly adsorbed onto GO, results in quenching of the fluorescent label and no fluorescence signal. Upon adding MUC1, it can recognize aptamer specifically and form aptamer/MUC1 complexes, resulting in the aptamer being away from GO. And then, when DNase I is introduced, enzymatic digestion reaction that releases the MUC1 for the next round occurs, leads to the accumulation a large amount of FAM fluorophores. The quantities of MUC1 can be achieved by fluorescence increment. Thus, a novel, sensitive, and specific aptasensor is obtained for assaying MUC1. Furthermore, it's successfully applied for detection of MUC1 in various spiked biological samples. One may believe that this sensing system possesses great potential for the monitoring of proteins, clinical diagnosis, and treatment.

\section{Experimental section}

\subsection{Reagents and materials}

The MUC1, EpCAM, BSA, PSA and VEGF were purchased from Cusabio Biotech Co. Ltd. (http://www.cusabio.cn/). Graphene oxide was purchased from XFNANO Co. Ltd. (Nanjing, China, http://www.xfnano.com). The deoxyribonuclease I (DNase I) were obtained from solarbio (Beijing, China, http:// www.solarbio.com). The MUC1 (5'-FAM-CCCGTCTTCCAGACAAGAGTGCAGGG-3') aptamer were synthesized by Sangon Biological Engineering Technology Co., Ltd. (Shanghai, China, http://www.sangon.com) and purified using high performance liquid chromatography. All of the reagents were diluted to the required concentration with working buffer $(10 \mathrm{mM}$ Tris, $100 \mathrm{mM} \mathrm{NaNO}$ pH 7.4) before usage. Healthy human serum, urine and saliva were provided by Affiliated Dongfeng Hospital, Hubei University of Medicine, and approved by Hospital's Ethics Committee. The other reagents employed were of analytical grade and used without further purification. Ultrapure water obtained from a Millipore water purification system (18.2 $\mathrm{M} \Omega \mathrm{cm}$ resistivity, Milli-Q Direct 8) was used in all runs.

\subsection{Optimization of sensing conditions}

Firstly, the 40 nM FAM labeled aptamer (probe) and a desired concentration of MUC1 was first mixed and kept at $37^{\circ} \mathrm{C}$ for $15 \mathrm{~min}$, followed by adding $15 \mu \mathrm{g} \mathrm{mL} \mathrm{m}^{-1}$ of GO. $5 \mathrm{~min}$ later, the reaction solution was added with $2 \mathrm{U} \mathrm{mL}^{-1}$ DNase I and incubated at $37^{\circ} \mathrm{C}$ for $15 \mathrm{~min}$, then the solution was diluted to $1 \mathrm{~mL}$. Finally, the fluorescence of the mixture were carried out on a Hitachi F-4600 spectrophotometer (Hitachi Co. Ltd, Japan, http://www.hitachi.co.jp) equipped with a xenon lamp excitation source at room temperature. The excitation was set at $495 \mathrm{~nm}$ and the emission spectra were collected from $510 \mathrm{~nm}$ to $600 \mathrm{~nm}$. The slits of the excitation and emission were both set at $5 \mathrm{~nm}$. The fluorescence intensity at $519 \mathrm{~nm}$ was used to choose the optimal experimental conditions and evaluate the performance of the proposed sensing system. In control experiments, the measurement process was all the same with the above except the addition of MUC1. Unless otherwise noted, each fluorescence measurement was repeated three times, and the standard deviation was plotted as the error bar.

\section{Results and discussion}

\subsection{Principle of design}

In the present study, the principle of fluorescent aptasensor detection of MUC1 based on DNase I-aided target recycling signal amplification is illustrated in Fig. 1. In the absence of MUC1, the GO can strongly adsorb single-stranded nucleic acids, thus, the MUC1 probe is adsorbed onto the surface of GO via $\pi-\pi$ stacking between DNA bases and GO, leading to the fluorescence is quenched, resulting in no fluorescence signal. Upon adding the MUC1, the probe sequences could be specific recognized by MUC1 to form probe/MUC1 complexes, leading to increase in the fluorescence intensity. Then, when the DNase I is added, all DNA sequences are degraded, resulting in the 


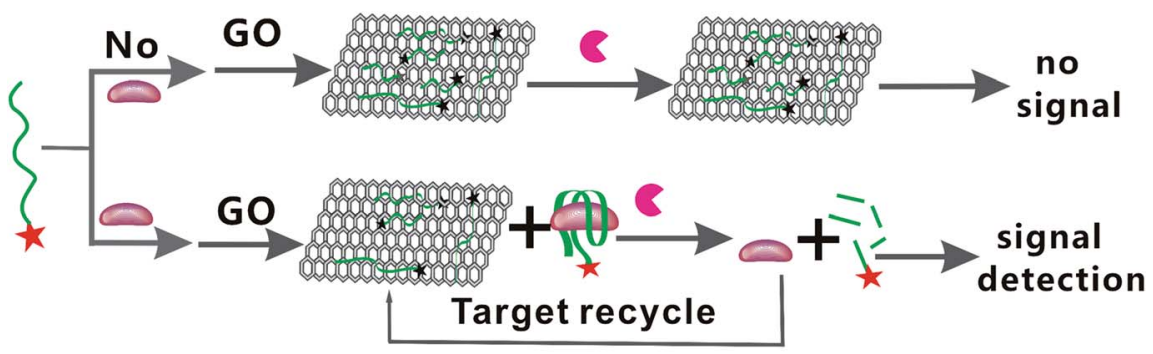

\section{MUC1 Dnase I * FAM}

MUC1 aptamer

Fig. 1 Schematic illustration of the GO-based fluorescent aptasensor assay detection of MUC1 by using DNase I-mediated target cyclic amplification.

release of MUC1 and FAM fluorophores. The released MUC1 is recycled repeatedly, leading to the accumulation of free FAM fluorophores. The quantities of MUC1 can be achieved by fluorescence increment. Thus, the fluorescence signal is significantly amplified by DNase I-mediated target recycling process.

\subsection{Feasibility analysis of the developed method for MUC1 detection}

To further verify the feasibility of DNase I-aided target recycling signal amplification strategy, Fig. 2 shows the fluorescence emission spectra under different conditions. The fluorescence signal produced by mixture solution DNase I and GO (curve c) is relatively weak in the absence of MUC1, indicating that GO can protect the probe from digesting by DNase I, resulting in the no FAM fluorophores are produced. And then, when the MUC1 is added, the fluorescence intensity do not obviously increase (curve b) in the absence of probe, thus, the MUC1 cannot specifically bind to probe, lead to the no FAM fluorophores are produced.

However, upon adding MUC1 and probe, significant enhancement of the fluorescence intensity was observed (curve a), as a result of the specific binding of probe to MUC1, lead to the probe/MUC1 complex formation, and keep the FAM

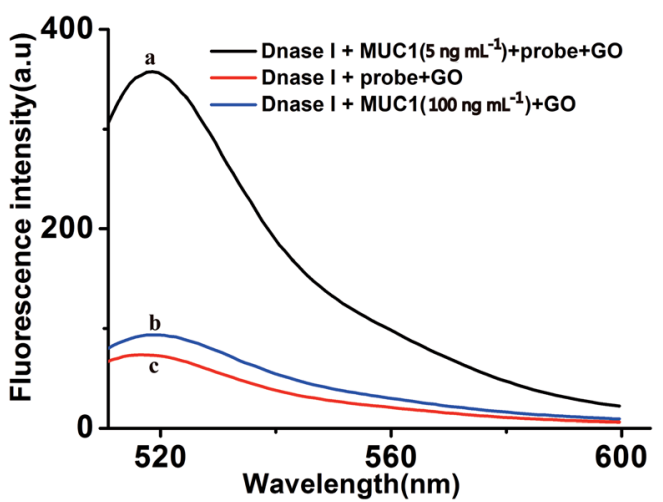

Fig. 2 The feasibility analysis. (a) Dnase I + MUC1 $\left(5 \mathrm{ng} \mathrm{mL}^{-1}\right)+$ probe + GO; (b) Dnase I + MUC1 (100 ng mL $\left.{ }^{-1}\right)+$ GO; (c) Dnase I + probe + $\mathrm{GO}$. The concentrations of Dnase I, GO and probe were $2 \mathrm{U} \mathrm{mL}^{-1}, 15$ $\mu \mathrm{g} \mathrm{mL} \mathrm{L}^{-1}$ and $40 \mathrm{nM}$, respectively. fluorophores away from GO. More importantly, the DNase Iaided target recycling results in the significant fluorescence amplification. Taking these results together, the feasibility of the proposed aptasensor for MUC1 detection by our design.

\subsection{Optimization of reaction conditions}

To achieve optimal sensing performance, several reaction conditions such as the concentration of DNase I, the concentration of $\mathrm{GO}$, the enzyme digestion time and reaction temperature were optimized. While the $F_{1}$ and $F_{0}$ were the fluorescence intensities in the presence and absence of MUC1, respectively. The fluorescence intensity and the value of $F_{1} / F_{0}$ are selected to evaluate the effects of the reaction conditions on the sensing performance of the method. As shown in Fig. 3A, Maximum $F_{1} / F_{0}$ value is observed when the concentration of DNase I was $2 \mathrm{U} \mathrm{mL}^{-1}$, however, $F_{1} / F_{0}$ value decreases obviously along with the further increasing of DNase I concentration. Thus, a DNase I concentration of $2 \mathrm{U} \mathrm{mL}^{-1}$ was confirmed as the optimized concentration. At the same time, the concentration of $\mathrm{GO}$ is another important factor affecting fluorescence intensity. As depicted in Fig. 3B, with the increase in the concentration of $\mathrm{GO}$, the fluorescence intensity initially increased and then gradually decrease, and maximum $F_{1} / F_{0}$ values is observed when the concentration of GO is $15 \mu \mathrm{g} \mathrm{mL}^{-1}$. Thus, $15 \mu \mathrm{g} \mathrm{mL} \mathrm{m}^{-1} \mathrm{GO}$ are used in the subsequent experiments.

In addition, the enzyme digestion time and reaction temperature are another important reaction condition affecting fluorescence intensity for this sensor. The fluorescence intensity and the value of $F_{1} / F_{0}$ change is related to the enzyme digestion reaction time. As shown in Fig. $4 \mathrm{~A}$, maximum $F_{1} / F_{0}$ values is observed when enzyme digestion time is $15 \mathrm{~min}$. Thus, 15 min of enzyme digestion time was selected for the rest of the experiments. Fig. 4B shows that the enzyme digestion temperature could obviously affect the sensitivity; the $F_{1} / F_{0}$ value reached a maximum when enzyme digestion temperature was $37{ }^{\circ} \mathrm{C}$ and then decreased gradually. Therefore, $37{ }^{\circ} \mathrm{C}$ was confirmed as the optimized enzyme digestion temperature.

To obtain high sensitivity, the concentrations of aptamer were also investigated. As shown in Fig. S1, $\dagger$ the concentration of aptamer was selected in the following experiments; the $F_{1} / F_{0}$ value reached a maximum when the concentration of aptamer 
A

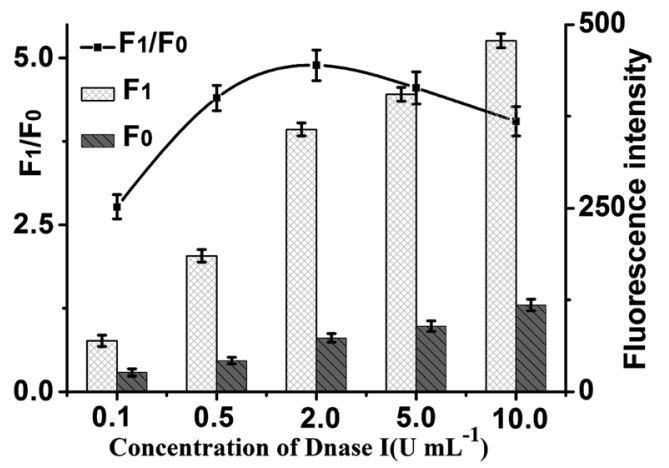

B

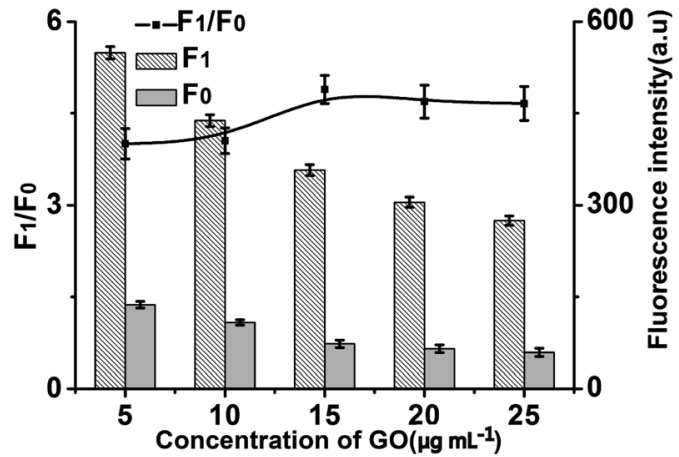

Fig. 3 (A) The effect of Dnase I concentration on the fluorescence response of this method. (B) The effect of GO concentration on the fluorescence response of this method. $F_{0}$ : control experiments; $F_{1}$ : with $5 \mathrm{ng} \mathrm{mL}{ }^{-1}$ of $\mathrm{MUC1}$, and the concentrations of probe is $40 \mathrm{nM}$. The black lines represent the $F_{1} / F_{0}$ at different conditions, while the $F_{1}$ and $F_{0}$ were the fluorescence intensities in the presence and absence of $M U C 1$, respectively. Error bars: SD, $n=3$.

A

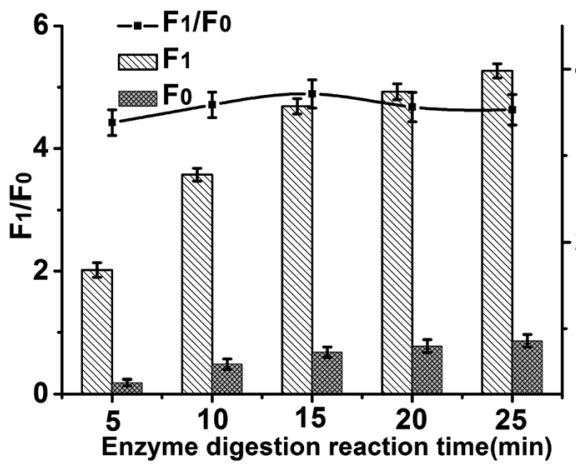

B

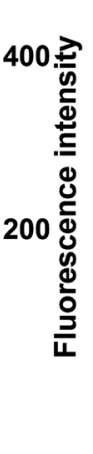

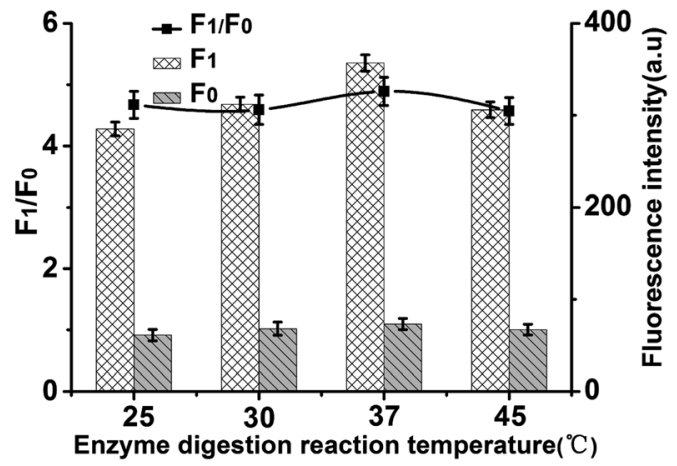

Fig. 4 The effect of enzyme digestion reaction time and reaction temperature on the fluorescence intensity at the emission wavelength of $519 \mathrm{~nm}$. The concentrations of Dnase I, MUC1, probe and GO were $2 \mathrm{U} \mathrm{mL}^{-1}, 5 \mathrm{ng} \mathrm{mL}^{-1}, 40 \mathrm{nM}$ and $15 \mu \mathrm{g} \mathrm{mL}{ }^{-1}$, respectively. Error bars: SD, $n=3$.

A

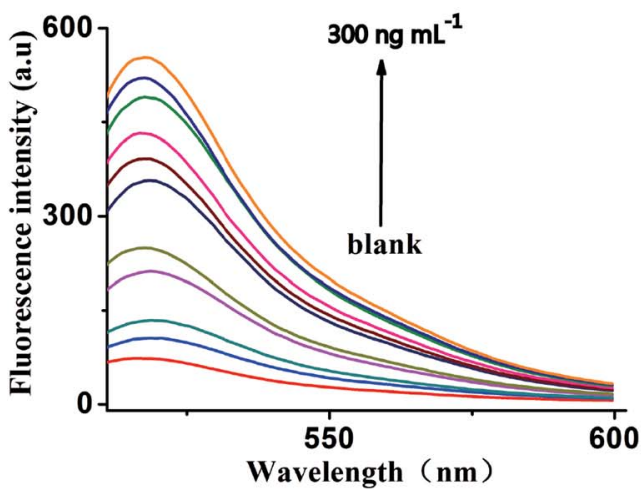

B

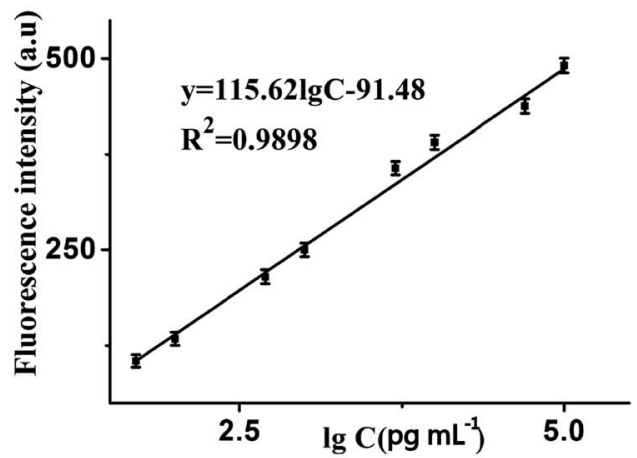

Fig. 5 (A) Fluorescence emission spectra of the biosensor in the presence of MUC1 with different concentrations: from bottom to top: 0,10 pg

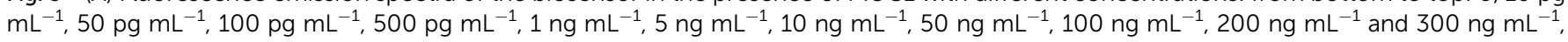
respectively. (B) The relationship curve of the fluorescence intensity as a function of MUC1 concentration. While shows the relationship between fluorescence intensity and MUC1 concentration, both experimental conditions: Dnase I, $2 \mathrm{U} \mathrm{mL}^{-1} ; \mathrm{MUC1}_{1} 5 \mathrm{ng} \mathrm{mL}{ }^{-1} ; \mathrm{probe}, 40 \mathrm{nM} ; \mathrm{GO}, 15 \mu \mathrm{g}$ $\mathrm{mL}^{-1}$ and the emission wavelength of $519 \mathrm{~nm}$. Error bars: SD, $n=3$. 
Table 1 The comparison of designed method for the detection of MUC1 with other reported biosensors

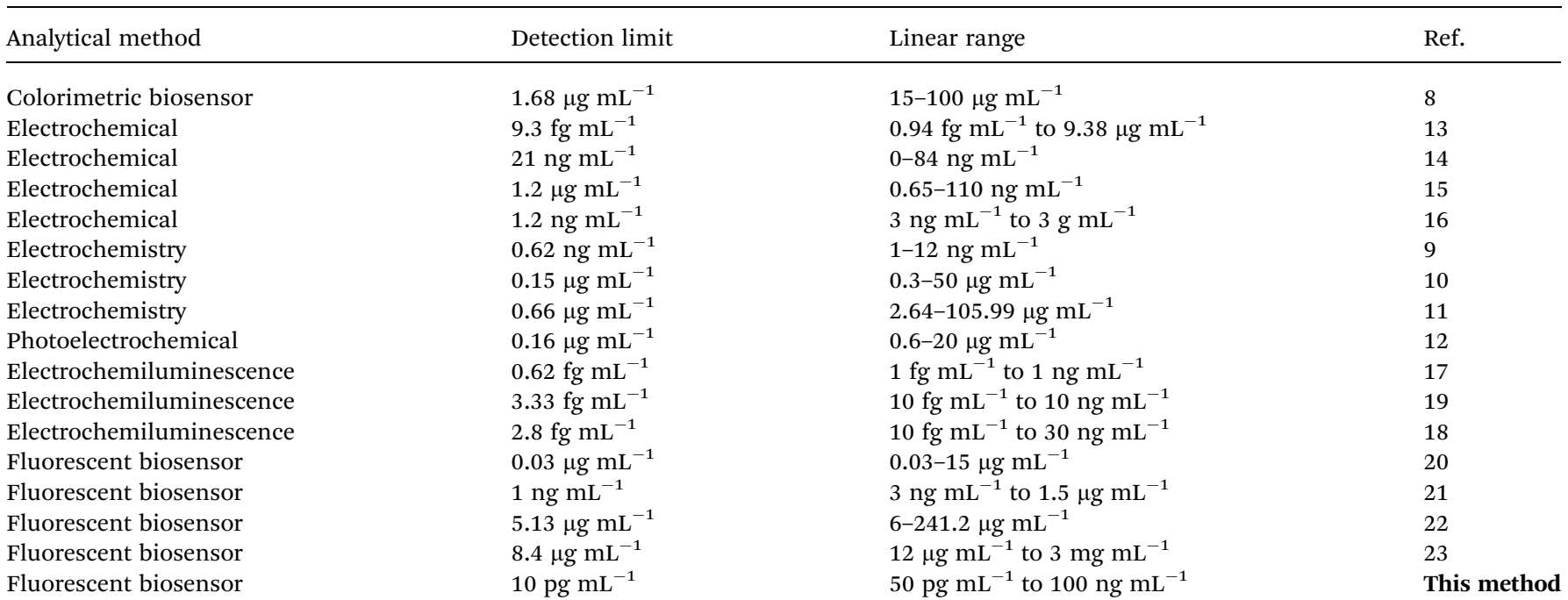

was $40 \mathrm{nM}$. Therefore, a concentration of $40 \mathrm{nM}$ was selected as the optimal concentration of aptamer.

\subsection{Sensitivity and specificity}

Under the optimal reaction conditions, the sensitivity of the sensor for detection of MUC1 is investigated. Fig. 5A shows the fluorescence emission spectra of the biosensor incubated in different concentrations of MUC1. We found that the fluorescence dramatically enhance with the increasing concentration of MUC1 from 0 to $300 \mathrm{ng} \mathrm{mL}^{-1}$. It illustrates a highly concentration dependence of the sensor for detection of MUC1. At the same time, Fig. 5B shows a good linear correlation between the fluorescence intensity and the concentration of MUC1 in the range from $50 \mathrm{pg} \mathrm{mL}^{-1}$ to $100 \mathrm{ng} \mathrm{mL} \mathrm{m}^{-1}$. The calibration plot of the linear equation is given as $y=115.62 \mathrm{lg} c$ - $91.48\left(R^{2}=0.9898\right)$, where $\lg c$ is logarithm of MUC1 concentration and $y$ is the fluorescence intensity. Furthermore,

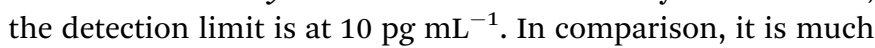

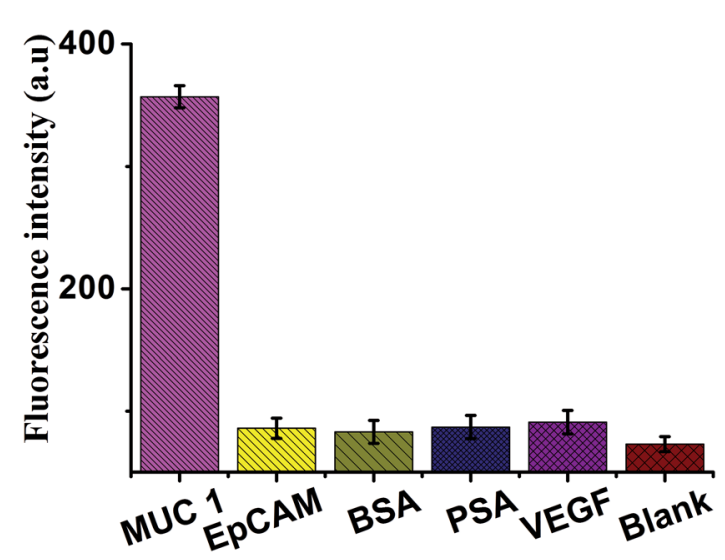

Fig. 6 Fluorescence intensity (at the emission wavelength of $519 \mathrm{~nm}$ ) of the sensor in the presence of MUC1 $\left(5 \mathrm{ng} \mathrm{mL}^{-1}\right)$, EpCAM (50 $\mathrm{ng}$ $\left.\mathrm{mL}^{-1}\right), \mathrm{BSA}\left(50 \mathrm{ng} \mathrm{mL}^{-1}\right), \operatorname{PSA}\left(50 \mathrm{ng} \mathrm{mL}^{-1}\right), \operatorname{VEGF}\left(50 \mathrm{ng} \mathrm{mL}^{-1}\right)$ and black, respectively. Error bars: SD, $n=3$. lower than other sensors reported in literatures listed in Table 1. In addition, for the specificity study, adding different control proteins was investigated, including EpCAM $\left(50 \mathrm{ng} \mathrm{mL}{ }^{-1}\right)$, BSA

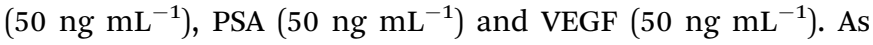
shown in Fig. 6, in the presence of other control proteins (50 ng $\left.\mathrm{mL}^{-1}\right)$, the significant increase of fluorescence signal is observed in the presence of the MUC1 ( $5 \mathrm{ng} \mathrm{mL}{ }^{-1}$ ), indicating that this proposed strategy exhibited good specificity for MUC1 detection.

\subsection{Determination of MUC1 in real samples}

To further verify the potential applicability of this present strategy in biological samples, the detection of MUC1 in biological samples by spiking MUC1 to human urine, saliva and serum diluted to $10 \%$ with buffer solution with the final concentration of $5 \mathrm{ng} \mathrm{mL}{ }^{-1}$ were performed. As shown in Fig. 7, a significant increase in various biological samples is observed, compared with no spiking biological samples. These results clearly demonstrate that this sensor can be a potential analytical method to detect MUC1 in real samples sensitively. The recoveries for the various concentrations of spiked MUC1 in

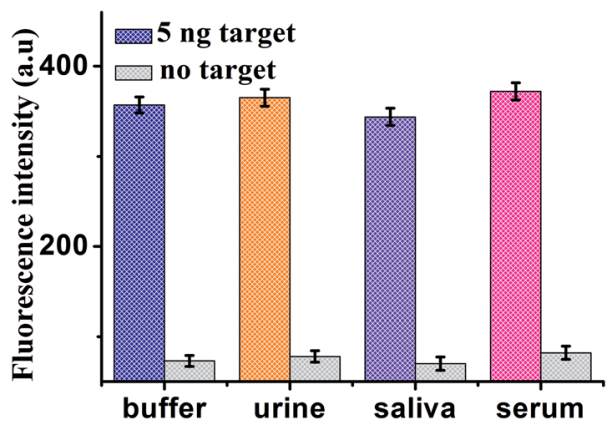

Fig. 7 Fluorescence intensity of the sensor for detection of MUC1 in buffer and blank biological samples (human urine, saliva and serum, respectively). Error bars: SD, $n=3$. 
Table 2 Recovery of MUC1 spiked in human serum samples

\begin{tabular}{lcccc}
\hline Serum sample & Added MUC1/ng & MUC1 found/nM & Recovery (100\%) \\
\hline 1 & 0 & 0 & -110.0 & RSD (\%) \\
2 & 0.10 & $0.11^{a}$ & 108.4 & 7.9 \\
3 & 5.0 & $5.42^{a}$ & 111.2 & 9.8 \\
4 & 50.0 & $55.6^{a}$ & & 8.9 \\
${ }^{a}$ & & &
\end{tabular}

human serum were in the range of $108.4-111.2 \%$, with the relative standard deviations (RSDs) of $7.9 \%, 9.8 \%$ and $8.9 \%$ at $0.1,5.0$ and $50.0 \mathrm{ng} \mathrm{mL} \mathrm{m}^{-1}$ of MUC1, respectively. This indicates an acceptable precision and reproducibility of the present approach for detecting MUC1 in real samples $(n=3)$ (Table 2).

\section{Conclusions}

In summary, we have successfully developed an amplified GObased fluorescence aptasensor for detecting MUC1. In this design, because of the deoxyribonuclease I-aided target recycling signal amplification, and based on GO efficient fluorescence quencher, selectivity and sensitivity for MUC1 were achieved. As to MUC1, this sensor can detect MUC1 with a linear range from $50 \mathrm{pg} \mathrm{mL}^{-1}$ to $100 \mathrm{ng} \mathrm{mL} \mathrm{m}^{-1}$, and the detection limit was low to $10 \mathrm{pg} \mathrm{mL}^{-1}$. In addition, the fluorescence signals are also obtained when this sensor is used for biological samples. More importantly, this biosensor achieved a high sensitivity by DNase I-aided target recycling and accumulated plenty of FAM fluorophores. Thereby, this design provides an efficient platform for MUC1 detection, may be a potential method for tumor diagnosis, bioanalysis, and clinical analysis.

\section{Conflicts of interest}

There are no conflicts to declare.

\section{Acknowledgements}

These works were supported by the National Natural Science Foundation of China (81872509), Hubei University of Medicine Free exploration Project (FDFR201804), the Science and Technology Hall of Hubei (No. 2013CFB469), the Natural Science Foundation of Hubei Provincial Department of Education (No. D20172101 and B2018435), the Science and Technology Key Program of Shiyan (No. 18Y101, $18 Y 82$ and 18Y87), the School Foundation for Hubei University of Medicine (No. FDFR201614), and the Key Discipline Project of Hubei University of Medicine.

\section{References}

1 R. Siegel, D. Naishadham and A. Jemal, Ca-Cancer J. Clin., 2010, 62, 10-29.

2 Q. Chen, D. Li, J. Ren, C. Li and Z. X. Xiao, Biochem. Biophys. Res. Commun., 2013, 440, 179-183.
3 S. Müller, K. Alving, J. Peterkatalinic, N. Zachara, A. A. Gooley and F. G. Hanisch, J. Biol. Chem., 1999, 274, 18165-18172.

4 A. K. H. Cheng, H. Su, Y. A. Wang and H. Z. Yu, Anal. Chem., 2009, 81, 6130-6139.

5 H. Cao, D. Ye, Q. Zhao, J. Luo, S. Zhang and J. Kong, Analyst, 2014, 139, 4917-4923.

6 F. Ma, C. Ho, A. K. H. Cheng and H. Z. Yu, Electrochim. Acta, 2013, 110, 139-145.

7 C. S. M. Ferreira, K. Papamichael, G. Guilbault, T. Schwarzacher, J. Gariepy and S. Missailidis, Anal. Bioanal. Chem., 2008, 390, 1039-1050.

8 S. Liu, N. Xu, C. Tan, W. Fang, Y. Tan and Y. Jiang, Anal. Chim. Acta, 2018, 1018, 86-93.

9 Z. Taleat, C. Cristea, G. Marrazza, M. Mazloum-Ardakani and R. Săndulescu, J. Electroanal. Chem., 2014, 717-718, 119-124. 10 Q. Guo, X. Li, C. Shen, S. Zhang, H. Qi, T. Li and M. Yang, Microchim. Acta, 2015, 182, 1483-1489.

11 R. Hu, W. Wen, Q. Wang, H. Xiong, X. Zhang, H. Gu and S. Wang, Biosens. Bioelectron., 2014, 53, 384-389.

12 J. Tian, T. Huang and J. Lu, Anal. Methods, 2016, 8, 23752382.

13 P. Gupta, A. Bharti, N. Kaur, S. Singh and N. Prabhakar, J. Electroanal. Chem., 2018, 813, 102-108.

14 A. Florea, A. Ravalli, C. Cristea, R. Săndulescu and G. Marrazza, Electroanalysis, 2015, 27, 1594-1601.

15 A. E. Karpik, B. P. Crulhas, C. B. Rodrigues, G. R. Castro and V. A. Pedrosa, Electroanalysis, 2017, 29, 1-9.

$16 \mathrm{~W}$. Wen, R. Hu, T. Bao, X. Zhang and S. Wang, Biosens. Bioelectron., 2015, 71, 13-17.

17 X. Jiang, H. Wang, H. Wang, Y. Zhuo, R. Yuan and Y. Chai, Anal. Chem., 2017, 89, 4280-4286.

18 X. Jiang, H. Wang, H. Wang, R. Yuan and Y. Chai, Anal. Chem., 2016, 88, 9243-9250.

19 F. Yang, X. Jiang, X. Zhong, S. Wei and R. Yuan, Sens. Actuators, B, 2018, 265, 126-133.

20 X. Liu, Y. Qin, C. Deng, J. Xiang and Y. Li, Talanta, 2015, 132, 150-154.

21 C. Ma, H. Liu, T. Tian, X. Song, J. Yu and M. Yan, Biosens. Bioelectron., 2016, 83, 15-18.

22 J. Lotif, Anal. Methods, 2015, 7, 7792-7798.

23 Y. He, Y. Lin, H. Tang and D. Pang, Nanoscale, 2012, 4, 20542059.

24 N. Ma, W. Jiang, T. Li, Z. Zhang, H. Qi and M. Yang, Microchim. Acta, 2015, 182, 443-447.

25 A. D. Ellington and J. W. Szostak, Nature, 1992, 355, 850-852.

26 C. Tuerk and L. Gold, Science, 1990, 249, 505-510.

27 T. Hermann and D. J. Patel, Science, 2000, 287, 820-825. 
28 M. Chen, S. Bi, X. Jia and P. He, Anal. Chim. Acta, 2014, 837, 44-51.

29 X. Liu, R. Aizen, R. Freeman, O. Yehezkeli and I. Willner, ACS Nano, 2012, 6, 3553-3563.

30 M. V. Berezovski, M. Lechmann, M. U. Musheev, T. W. Mak and S. N. Krylov, J. Am. Chem. Soc., 2008, 130, 9137-9143.

31 M. A. Tabrizi and M. Shamsipur, Biosens. Bioelectron., 2015, 74, 764-769.

32 J. Chen, B. Shang, H. Zhang, Z. Zhu, L. Chen, H. Wang, F. Ran, Q. Chen and J. Chen, RSC Adv., 2018, 8, 14798-14805.

33 X. Yan, W. Li, K. Liu and L. Deng, Anal. Methods, 2015, 7, 10243-10250.

34 Y. Tang, F. Long, C. Gu, C. Wang, S. Han and M. He, Anal. Chim. Acta, 2016, 933, 182-188.

35 P. Gupta, A. Bharti, N. Kaur, S. Singh and N. Prabhakar, J. Electroanal. Chem., 2018, 813, 102-108.

36 D. A. Dikin, S. Stankovich, E. J. Zimney, R. D. Piner, G. H. Dommett, G. Evmenenko, S. T. Nguyen and R. S. Ruoff, Nature, 2007, 448, 457-460.
37 Z. Liu, J. T. Robinson, X. Sun and H. Dai, J. Am. Chem. Soc., 2008, 130, 10876-10877.

38 K. Liu, X. Yan, B. Mao, S. Wang and L. Deng, Microchim. Acta, 2016, 183, 643-649.

39 Y. Wang, Z. Li, J. Wang, J. Li and Y. Lin, Trends Biotechnol., 2011, 29, 205-212.

40 M. L. Chen, Y. J. He, X. W. Chen and J. H. Wang, Bioconjugate Chem., 2013, 24, 387-397.

41 B. Liu, Z. Sun, X. Zhang and J. Liu, Anal. Chem., 2013, 85, 7987-7993.

42 Y. Ning, Y. Duan, Y. Feng and L. Deng, Anal. Lett., 2014, 47, 2350-2360.

43 Q. He, H. Luo, L. Tang, J. Liu, K. Chen, Q. Zhang and Y. Ning, Microchim. Acta, 2017, 184, 3875-3882.

44 Y. Xie, X. Lin, Y. Huang, R. Pan, Z. Zhu, L. Zhou and C. J. Yang, Chem. Commun., 2015, 51, 2156-2158. 